\title{
Socioeconomic status and vaccine coverage during wild-type poliovirus emergence in Israel
}

\author{
B. BINYAMINY ${ }^{1}$, N. BILENKO ${ }^{2,3}$, E. J. HAAS ${ }^{3,4}$, I. GROTTO ${ }^{2,5}$ AND \\ M. GDALEVICH ${ }^{3,6}$ \\ ${ }^{1}$ Edith Wolfson Medical Center, Directorate, Holon, Israel \\ ${ }^{2}$ Department of Public Health, Faculty of Health Sciences, Ben-Gurion University of the Negev, Beer-Sheva, \\ Israel \\ ${ }^{3}$ Regional Office of Ministry of Health, Southern District, Beer-Sheva, Israel \\ ${ }^{4}$ Department of Pediatrics, Faculty of Health Sciences, Ben-Gurion University of the Negev, Beer-Sheva, Israel \\ ${ }^{5}$ Public Health Services, Ministry of Health, Jerusalem, Israel \\ ${ }^{6}$ Division of Community Health, Faculty of Health Sciences, Ben-Gurion University of the Negev, Beer-Sheva, \\ Israel
}

Received 8 October 2015; Final revision 4 February 2016; Accepted 14 April 2016;

first published online 4 May 2016

\section{SUMMARY}

In August 2013, a nationwide vaccination campaign with bivalent oral polio vaccine (bOPV) was initiated after isolation of wild-type poliovirus type 1 (WPV-1) in routine sewage surveillance in Israel. The campaign started in the Southern district and later extended to the entire country. This study examined the association between socioeconomic status (SES), and compliance with bOPV vaccine during the campaign. Nationwide data relating to SES by geographical cluster were correlated with vaccine coverage rates in the same areas. All analyses were conducted separately for Jews and Arabs. Coverage with the bOPV vaccination campaign in the Arab population $(92 \cdot 4 \%)$ was higher than in the Jewish population $(59 \cdot 2 \%)$. This difference was consistently present in all SES clusters. In the Jewish population there was an inverse correlation between SES and vaccination coverage rates $(R=-0 \cdot 93, P<0 \cdot 001)$. Lower vaccination coverage with supplemental vaccine activities in higher SES groups is a challenge that needs to be addressed in future public health events and emergencies in order to achieve satisfactory protection rates for the public.

Key words: Public health, polio, vaccination (immunization), vaccine policy development.

\section{INTRODUCTION}

Poliomyelitis has been targeted for eradication by the World Health Organization (WHO) since 1988. The intended timeline for the completion of eradication was targeted for the end of 2000 [1]. The European

\footnotetext{
* Author for correspondence: Dr B. Binyaminy, Edith Wolfson Medical Center, Directorate, 62 Halohamin St, P.O.B. 5, Holon 58100-15, Israel.

(Email: binyaminb@wolfson.health.gov.il)
}

Region of the WHO, of which Israel is a member, was declared polio-free in 2002 [2]. As of 2015, only two countries remained endemic for wild-type poliovirus infection: Afghanistan and Pakistan. Many other countries are experiencing the re-introduction of poliovirus, e.g. Equatorial Guinea, Ethiopia, Somalia, Cameroon, Iraq, Nigeria and Syrian Arab Republic [3], complicating eradication efforts.

In Israel, the last known transmission of poliovirus occurred in 1988, resulting in 15 paralytic cases [4]. A nationwide vaccination campaign was initiated, and 
over $90 \%$ of the target population (aged 2 months -40 years) was immunized to contain the event [5]. Between 1990 and 2004, the national childhood vaccination plan included three doses of inactivated polio vaccine (IPV) and three doses of live-trivalent attenuated oral polio vaccine (OPV) during the first 15 months of life. An additional OPV booster dose was given during first school year [6]. Concurrently, routine monitoring of wild polioviruses (WPV) was initiated, including an environmental polio surveillance programme through monthly sampling of 8-10 sentinel sewage treatment facilities and mandatory notification of clinical cases of acute flaccid paralysis (AFP) in children up to age 15 years [7]. In 2005, 3 years after the WHO declaration of the European Region, including Israel, as polio free [2], the Ministry of Health (MoH) ceased using OPV. The subsequent vaccination approach included a five-dose IPV schedule, given at ages 2, 4, 6, and 12 months with a booster dose at second school year [5]. The most recent IPV national coverage (fourth dose added at age 2 years) rates in Israel are estimated at $95 \%[8,9]$.

After a long polio-free period (last poliomyelitis case in 1988), in May 2013, viral isolates from environmental sewage samples from Rahat, a town in the Southern district of the country, were identified as WPV type 1 [7]. The findings recurred in sequential samples in several other communities in Southern Israel. The $\mathrm{MoH}$ responded to these findings by expanding the number of localities performing sewage sampling and by increasing the frequency of sampling. A national IPV catch-up vaccination campaign in children aged up to 6 years was initiated, a health promotion campaign emphasizing the importance of hand washing was advanced through the media and enhanced active surveillance of cases of AFP was broadened to include cases of aseptic meningitis [7].

Despite these efforts, environmental surveillance samples continued to be positive for WPV-1. The evidence of re-introduction of poliovirus into the community was strengthened by identification of WPV-1 in consecutive stool samples collected from healthy children and adults [6]. Since the immune protection of children born after 2004 is based solely on high coverage with IPV, a large cohort of children had no intestinal immunity which could lead to persistence of wild virus circulation [8]. Therefore, a broad supplementary vaccination campaign with bivalent OPV (bOPV) for children born after 1 January 2004 who received at least one dose of IPV was initiated on 5
August 2013 in the Southern district. Shortly after the detection of WPV-1 in sewage samples from other areas, the campaign was extended to the whole country. The vaccination campaign that included administration of one bOPV dose continued until December 2013. Since no cases of paralytic polio were detected, the major goal of the vaccination campaign was to ensure, as efficiently and rapidly as possible, the cessation of poliovirus circulation in the country.

Children aged $<10$ years were defined as the target group for bOPV, because they had not previously received OPV and were not expected to have adequate intestinal immunity, putting them at risk of transmitting the virus to other unvaccinated or under-vaccinated persons.

A major effort was invested in increasing vaccination coverage. Assessment of population compliance trends and understanding related factors in this setting is of major importance. Some of these factors, including the logistic structure of the vaccine delivery system and communication to the public [10], were similar throughout the country. However, other population characteristics and parental attitudes towards the disease and vaccines [11] may vary by location or be based on other characteristics. Studies attempting to demonstrate the association between socioeconomic status (SES) and vaccines have shown conflicting results [10-14].

In Israel, this association is further complicated due to existing ethnic differences. The country includes two main ethnic groups: Jews and Arabs, comprising $75 \%$ and $21 \%$ of the population, respectively [15], and a significant disparity in SES as well as health indicators exists between these two subgroups. Sixty-eight per cent and 23\% of children in the Arab and Jewish population, respectively, live in conditions of poverty [16]. There are 2.5 times more Jews with at least 16 years of education than Arabs [16].

In this study we assessed the association between compliance with oral polio vaccination during the described event in Israel and SES, stratified by ethnicity.

\section{METHODS}

This is a population-based study, including the entire population of Israel aged $<10$ years. Vaccine coverage was calculated by dividing the number of all the vaccinees by the number of candidates. Since the $\mathrm{MoH}$ invested significant efforts to maximize vaccine coverage by using a communication campaign through the 
Table 1. Vaccine coverage, nationwide and by district, for Jewish, Arabic, and mixed communities

\begin{tabular}{|c|c|c|c|c|c|c|c|c|c|}
\hline \multirow[b]{2}{*}{ District } & \multicolumn{3}{|l|}{ Jews } & \multicolumn{3}{|l|}{ Arabs } & \multicolumn{3}{|l|}{ Mixed } \\
\hline & Targeted $(n)$ & Vaccinated $(n)$ & $\%$ & Targeted $(n)$ & Vaccinated $(n)$ & $\%$ & Targeted $(n)$ & Vaccinated $(n)$ & $\%$ \\
\hline General & 895618 & 529965 & $59 \cdot 2$ & 244632 & 225993 & $92 \cdot 4$ & 241567 & 153892 & $63 \cdot 7$ \\
\hline South & 70997 & 48562 & $68 \cdot 4$ & 60973 & 54532 & $89 \cdot 4$ & & & \\
\hline North & 83722 & 51019 & $60 \cdot 9$ & 119899 & 112107 & $93 \cdot 5$ & 14577 & 9900 & $67 \cdot 9$ \\
\hline Central & 331569 & 185835 & 56 & 23134 & 21403 & $92 \cdot 5$ & 23845 & 18722 & $78 \cdot 5$ \\
\hline Haifa & 63690 & 37255 & $58 \cdot 5$ & 38423 & 36117 & 94 & 32383 & 18908 & $58 \cdot 4$ \\
\hline Ashkelon & 79509 & 53077 & $66 \cdot 8$ & 33 & 31 & $93 \cdot 9$ & & & \\
\hline Jerusalem & 75300 & 47747 & $63 \cdot 4$ & 2170 & 1803 & $83 \cdot 1$ & 170762 & 106362 & $62 \cdot 3$ \\
\hline Tel-Aviv & 190831 & 106470 & $55 \cdot 8$ & & & & & & \\
\hline
\end{tabular}

media and reaching out to parents of unvaccinated children, we designated non-vaccinated individuals as refusals. Therefore vaccine coverage is used as an approximation of compliance.

All children born after 1 January 2004 (aged 2 months -9 years), who received at least one dose of IPV (about 1300000 [17]), were included in the target population for the campaign. All of Israel's $1187 \mathrm{mu}-$ nicipalities, grouped into seven geographical districts (South, North, Center, Haifa, Ashkelon, Jerusalem, Tel-Aviv), were included. For each child arriving at the vaccination clinic, a questionnaire was completed order to rule out contraindications and the date and lot number of the vaccine were entered into the national computerized childhood vaccination database, which is used routinely for childhood vaccinations in the country.

Data were collected about the per cent coverage of bOPV (types 1 and 3) in each community following the national vaccination campaign [17]. For each community, the rating of SES was obtained from Israel's National Central Bureau of Statistics (CBS), based on demography, education, employment and standard-of-living variables [18]. The SES information was presented on an ordinal scale of 1-10 in accordance with the CBS definitions for the municipalities throughout the country. All of the municipalities were categorized into clusters based on these definitions and an additional cluster, 0, was added for the dispersed Bedouin population that has an undefined status, yet due to living in non-established settlements, known for especially low socioeconomic conditions.

Given substantial differences in health-related knowledge, attitudes, and behaviour in Israel's Jewish and Arab population [19, 20], the analysis was stratified by ethnicity. An ethnicity variable was defined, classifying each municipality as composed primarily (>90\% according to the CBS data) of Jewish residents, Arab residents or mixed.

\section{Statistical analysis}

Immunization coverage for each municipality was calculated as percentage of the target population. We examined the association between immunization coverage rate and SES scores by statistical measures: (1) Spearman correlation; (2) comparison between immunization coverage of two sets of groups, i.e. population in clusters $0-5$ vs. 6-10; population in clusters 0-2 vs. 8-10 using relative risk (RR) with $95 \%$ confidence interval (CI). Data were analysed using IBM SPSS software v. 21.0 (IBM Corp., USA). $P$ values $<0.05$ were considered statistically significant.

As this was an analysis of data published on the Israel $\mathrm{MoH}$ website, ethical approval was not required.

\section{RESULTS}

The overall nationwide vaccine coverage in the campaign conducted in children aged 2 months -9 years was $66 \%$ [17], while in Jewish subgroups coverage was $59 \%$, and in Arab subgroups coverage was $92 \%$. Differences were observed in vaccine coverage between the seven districts, where the highest coverage was in Southern and Northern districts (over 80\%) and the lowest in Tel-Aviv district (56\%) (Table 1). The vaccine coverage rates were consistently higher in the Arab population, across all SES strata.

\section{Spearman correlation analysis}

A negative correlation between vaccine coverage and SES was demonstrated in the nationwide data $\left(r_{s}=-0 \cdot 95, P<0 \cdot 0001\right)$ (Fig. 1 and Table 2). The 


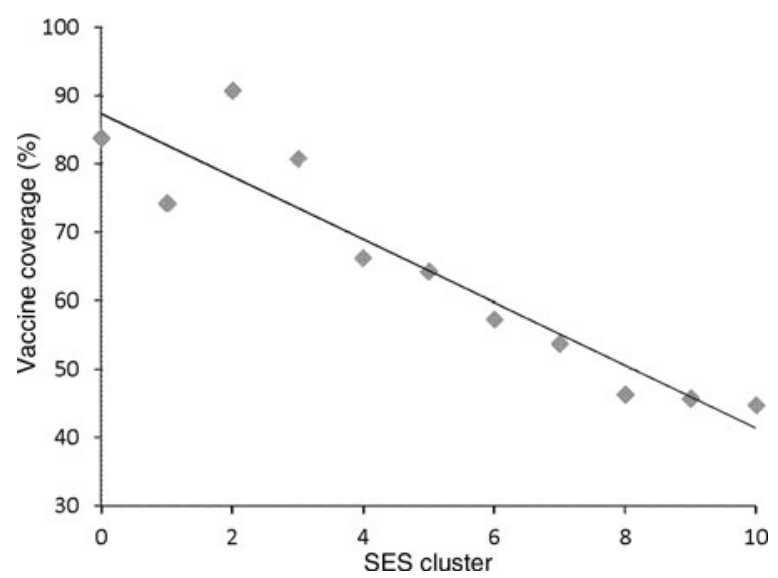

Fig. 1. The correlation between bivalent oral polio vaccine coverage and socioeconomic status (SES) cluster. A SES cluster of 0 refers to dispersed Bedouin communities that are not recognized municipalities.

Table 2. Vaccine coverage stratified by socioeconomic status score

\begin{tabular}{lcccc}
\hline \hline & \multicolumn{4}{l}{ Total } \\
\cline { 2 - 5 } Score & Proportion (\%) & Targeted $(n)$ & Vaccinated $(n)$ & $\%$ \\
\hline $0^{*}$ & $1 \cdot 4$ & 19250 & 16143 & $83 \cdot 9$ \\
1 & $4 \cdot 1$ & 56767 & 42163 & $74 \cdot 3$ \\
2 & $10 \cdot 2$ & 140168 & 127223 & $90 \cdot 8$ \\
3 & $11 \cdot 1$ & 153127 & 123750 & $80 \cdot 8$ \\
4 & $18 \cdot 8$ & 259212 & 171866 & $66 \cdot 3$ \\
5 & $21 \cdot 7$ & 299522 & 192564 & $64 \cdot 3$ \\
6 & $11 \cdot 5$ & 158945 & 91284 & $57 \cdot 4$ \\
7 & $7 \cdot 2$ & 99391 & 53506 & $53 \cdot 8$ \\
8 & $12 \cdot 9$ & 178054 & 82641 & $46 \cdot 4$ \\
9 & $0 \cdot 9$ & 12977 & 5936 & $45 \cdot 7$ \\
10 & $0 \cdot 1$ & 1779 & 797 & $44 \cdot 8$ \\
Total & 100 & 1379192 & 907873 & $65 \cdot 8$ \\
\hline \hline
\end{tabular}

* A score of 0 refers to dispersed Bedouin communities that are not recognized municipalities.

trend was more obvious between SES clusters 4-8 which include more than $70 \%$ of the population $\left(r_{s}\right.$ $=-1, P<0 \cdot 0001)$. According to the correlation coefficient, $91 \%$ of the coverage variance is explained by SES. Separate analyses for nationwide correlations between SES and vaccine coverage, stratified by ethnicity, showed that the inverse association was present in Jewish communities $\left(r_{s}=-0.93, P<0.0005\right)$, yet absent in the Arab sector $\left(r_{s}=0.33, P=0.38\right)$ (Fig. 2 and Table 3). Again, the trend in the Jewish population is more obvious between SES clusters 4-8 which include more than $80 \%$ of the Jewish population $\left(r_{s}=-1, P<0.0001\right)$.

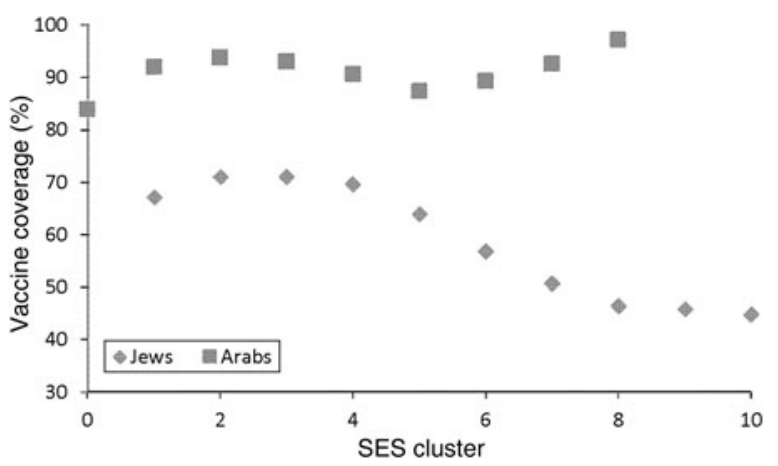

Fig. 2. The correlation between bivalent oral polio vaccine coverage and socioeconomic status (SES) cluster stratified by Jewish or Arabic ethnicity. Mixed Arabic and Jewish municipalities are not included in the figure. An SES cluster of 0 refers to dispersed Bedouin communities that are not recognized municipalities.

The association between coverage and SES varied in the two ethnic populations (Fig. 2). While vaccination rates were similar across all SES strata in the Arab subgroup, in the Jewish population they were higher in the lower SES groups. Therefore no combined analysis was performed.

\section{Receipt of vaccine in low and high SES groups by ethnicity}

Jews in lower SES clusters (0-5) were more like to have received vaccine than Jews in higher SES clusters (6-10) (RR 1·30, 95\% CI 1·29-1·31). This difference was accentuated when comparing the lowest SES clusters (0-2) to the highest SES clusters (8-10) (RR 1.47, 95\% CI 1.46-1.48). However, no significant differences were found in the Arab population for lower and higher clusters (RR 1.02, 99\% CI 1.01-1.03). In addition, comparison of the lowest and highest SES clusters of Arabic towns showed no difference between clusters (RR 0.95, 95\% CI 0.9-1.0) (see Table 4).

\section{DISCUSSION}

Our results demonstrate an inverse association between socioeconomic status and bOPV coverage in the Jewish population, as lower SES groups tended towards higher coverage. Given an extensive media and publicity effort, it is unlikely that there was lack of awareness of the vaccination campaign, especially not in high SES groups. Moreover, there is greater accessibility to healthcare services in high socioeconomic municipalities which rules out lack of access as a 
Table 3. Vaccine coverage stratified by socioeconomic status score and ethnicity

\begin{tabular}{|c|c|c|c|c|c|c|c|c|c|}
\hline \multirow[b]{2}{*}{ Score } & \multicolumn{3}{|l|}{ Jews } & \multicolumn{3}{|l|}{ Arabs } & \multicolumn{3}{|l|}{ Mixed } \\
\hline & Targeted $(n)$ & Vaccinated $(n)$ & $\%$ & Targeted $(n)$ & Vaccinated $(n)$ & $\%$ & Targeted $(n)$ & Vaccinated $(n)$ & $\%$ \\
\hline $0^{*}$ & & & & 19250 & 16017 & $83 \cdot 2$ & & & \\
\hline 1 & 40330 & 26821 & $66 \cdot 5$ & 35717 & 31026 & $86 \cdot 9$ & & & \\
\hline 2 & 18371 & 12951 & $70 \cdot 5$ & 121797 & 113574 & $93 \cdot 2$ & & & \\
\hline 3 & 84949 & 59616 & $70 \cdot 2$ & 68178 & 63124 & 92.6 & & & \\
\hline 4 & 48190 & 33335 & $69 \cdot 2$ & 9623 & 8666 & $90 \cdot 1$ & 201399 & 128309 & $63 \cdot 7$ \\
\hline 5 & 288515 & 182973 & $63 \cdot 4$ & 3268 & 2836 & $86 \cdot 8$ & 7739 & 5300 & $68 \cdot 5$ \\
\hline 6 & 156059 & 87975 & $56 \cdot 4$ & 2886 & 2561 & $88 \cdot 7$ & & & \\
\hline 7 & 65498 & 32883 & $50 \cdot 2$ & 1543 & 1423 & $92 \cdot 2$ & 32383 & 18766 & 58 \\
\hline 8 & 178054 & 80352 & $45 \cdot 1$ & & & & & & \\
\hline 9 & 12977 & 5875 & $45 \cdot 3$ & & & & & & \\
\hline 10 & 1779 & 796 & $44 \cdot 7$ & & & & & & \\
\hline
\end{tabular}

* A score of 0 refers to dispersed Bedouin communities that are not recognized municipalities.

Table 4. Likelihood of vaccination by SES clusters

\begin{tabular}{lll}
\hline \hline & SES clusters 0-5 vs. 6-10 & SES clusters 0-2 vs. 8-10 \\
\hline Jews & $1 \cdot 3(1 \cdot 29-1 \cdot 31)$ & $1.47(1 \cdot 46-1 \cdot 48)$ \\
Arabs & $1.02(1.01-1 \cdot 03)$ & $0.95(0 \cdot 9-1 \cdot 0)$ \\
\hline \hline
\end{tabular}

SES, Socioeconomic status.

Values presented as relative risk with $95 \%$ confidence interval.

reasonable explanation for lower vaccine coverage. Therefore, a difference in compliance is a probable explanation. Unlike previous experience where ultraorthodox Jewish communities sometimes resist vaccination, as shown in outbreaks of measles [21], most of them live in municipalities with low SES clusters (1-4) [22] and vaccine coverage was higher than for average Jewish subgroups ( $70 \%$ vs. $59 \%)$. The same outcome holds true for the Arab-Bedouin population. If the publicity message to vaccinate children did not reach a proportion of the population, we would expect them to be from the lower SES subgroups, as well as socially distanced subgroups (i.e. the Bedouin or the ultra-orthodox Jews). Yet the results point to a very high coverage in these subgroups and the lowest coverage rates were measured in the most mediaminded and socially attentive populations of the high SES subgroups, mostly Jewish subgroups.

The inverse association was not present in the Israeli Arab population, where vaccination compliance was consistently higher across all SES strata.

The differences in coverage between Arabs and Jews could be related to the dynamics of the 2013 polio event in Israel. WPV was first identified in the sewage systems in Arab municipalities in southern Israel and further surveillance showed higher concentrations of WPV-1 and higher prevalence in the Arab towns and villages. Furthermore, poliovirus circulation in this population continued for a longer period than elsewhere in the country. Consequently, efforts for disease prevention were primarily directed towards this population and were strengthened both in investment of public health resources as well as the reflection of the event in the media and public opinion.

Despite existing differences in socioeconomic parameters between the two main ethnic groups, Arabs and Jews, the routine childhood vaccination coverage in both groups is similar, averaging $95 \%[9,23]$. Given this high acceptance of vaccinations in all segments of Israeli society, the $<90 \%$ coverage during the polio event, portrayed in the media as a potential public health emergency, was surprising. It is possible that some parents perceived bOPV for their children as unnecessary because they were fully protected by prior vaccination with IPV.

Several studies including a systematic review of studies from low- and middle-income countries indicated a direct correlation between low SES and low immunization coverage [10-12]. Other studies have shown conflicting results. A study conducted in the United States which aimed to identify the characteristics of vaccine refusers found they were more likely to come from well-educated and higher-income areas [13]. Another study from the United States, that differentiated between under-vaccinated children and unvaccinated children [14], showed that under-vaccinated 
children tended to be of African-American ethnicity, to live in a single-parent home, to not have a college degree, and to live in a household near poverty level. Unvaccinated children tended to be white, to have a mother who was married and had a college degree, and to live in a household with high annual income. Other specific factors, like healthcare delivery, may be responsible for under-vaccination, especially in middleand low-income countries [11], whereas parental preference is more dominant in high-income countries [13, 14]. Parental attitude towards vaccination has been assessed in previous studies in Israel. Metzger et al. found that mothers who opposed hepatitis B vaccination (HBV) were more likely to have higher SES than mothers who did not oppose the vaccine [24]. In that study, healthcare professionals were also more likely to oppose HBV of their children. Analysis of the motivations for parents to oppose vaccination of their children against hepatitis B suggested that parents who refused to vaccinate had a tendency to value individualism/autonomy.

Studies have suggested cultural differences in willingness to act with respect to individual vs. collective interests, with Western culture tending to individualism whereas Asian and African culture tended to collectivism $[25,26]$. In this regard, Israel represents a middle ground where European and Asian or African tendencies may be brought forward within the context of one limited geographical location. The findings concerning the difference in coverage rates between Arab and Jewish sectors, irrespective of SES, may point to the similar reasoning of collectivism $v s$. individualism.

Other studies have shown that persons from higher SES groups tend to be more individualist whereas persons of lower SES tend to focus on the collective $[27,28]$. These models of social orientation have been used to describe willingness to vaccinate and differentiate between 'vaccine believers' and 'vaccine sceptics' with multiple gradations between the two extremes [29]. A study conducted by Velan et al. in Israel investigated attitudes in the public's response to vaccination and found three major attitude traits: acceptance, individualism, and differentiation [30]. These attitude traits were correlated with actual compliance with vaccination programmes. Moreover, attitudes differ significantly between Arabs and Jews (a higher per cent of Arabs were acceptors whereas a higher per cent of Jews were individualists) [30].

Divergent attitudes in the public during the bOPV campaign could explain why some parents perceived the bOPV as a 'societal' vaccine which aimed to protect the unvaccinated 'at the expense' of their IPV-vaccinated children for whom the vaccine is not necessary [31]. These parents may not be against vaccination generally, but they thought that their children had little to gain and on the other hand might suffer adverse effects from the vaccine. This may explain why according to the survey by the Government Advertising Bureau only $55 \%$ of parents said they would vaccinate their children although $80 \%$ said they believed $\mathrm{MoH}$ action was necessary as quoted by Kaliner et al. [31].

There are three main categories of motivation that may explain lack of willingness to vaccinate [32]: religious or philosophical objections to vaccination, preference for allowing the rest of the group to achieve the desired benefit ('free riders') and divergent risk perception of disease and vaccine risks and benefits. The silent re-introduction of the polio virus after a long period of no transmission was a new situation for Israeli society as well as for public health professionals in Israel. As the children whose parents decided not vaccinate them with bOPV were previously immunized with IPV and were protected from poliomyelitis, they cannot be classified as free riders. Since most of them were vaccinated with IPV against polio and thought that $\mathrm{MoH}$ action was justified, but not for their own children, they cannot be classified as the third category either. Thus, a potential fourth category of vaccine refusal comes to mind, i.e. people who respond negatively to the phenomenon of 'free riders' might not consent to vaccination for their children in order to protect those 'free riders'. However, because lower coverage of bOPV could enable ongoing poliovirus transmission in the community, reliance on IPV alone might not be the appropriate response on the national or global level, and the importance of the MoH's efforts in the larger picture may not have been appropriately emphasized or understood.

Our results, showing an inverse association between SES and vaccination compliance, support such a view of individualism in the Jewish sector of the country. Higher SES can be viewed as a construct proxy of factors, such as education, income and social status that may reflect individualistic tendencies. Considering these factors as part of causative pathway for individualism requires further research.

It is worth mentioning, that the bOPV campaign succeeded in terminating the transmission and excreting of WPV according to environmental surveillance 
despite lower coverage than in the routine immunization schedule. Since 2014 two supplementary doses of bOPV have been added to the immunization schedule as long as there was a potential threat of reintroduction of the virus in the region (e.g. Syria) [8].

The main strength of this study is its full population basis, overcoming selection bias. The national childhood vaccination computerized database is linked to the birth database and the possibility of omission is very low. Nevertheless, this was a population ecological study, based on national vaccination coverage data, employing geocoding (the name of the settlement) to assign ethnicity and SES. Since our study was not a survey, personal data for Israeli children aged $<10$ years, vaccination status, and SES were not available, leading to possible misclassification. Moreover, we were unable to determine the reasons for not vaccinating, and individual choice may have played a more important role than SES itself. An ideal approach to the study question, identifying individual determinants of willingness to vaccinate, was precluded within the context of a rapid vaccination campaign in a situation which was perceived by decision-makers as a national emergency.

\section{CONCLUSION}

There was an inverse correlation between SES and compliance to bOPV in the Jewish population. Additional findings show that the coverage of the bOPV national vaccine campaign varied substantially between different populations - higher in Arabs than in Jews. Recognition of the association of vaccine compliance with SES is important for policy-makers while considering the optimal approach for future outbreaks, especially possible polio events that may occur in Western countries where considerable ethnic variability and SES disparities exist.

\section{ACKNOWLEDGEMENTS}

This research received no specific grant from any funding agency, commercial or not-for-profit sectors.

\section{DECLARATION OF INTEREST}

None.

\section{REFERENCES}

1. World Health Organization. Forty-first health assembly. Global eradication of poliomyelitis by the year 2000 (http://www.who.int/ihr/polioresolution4128en.pdf), May 1988. Accessed 15 May 2014.

2. European Regional Certification Commission. Certification of poliomyelitis eradication. World Health Organization (http://www.euro.who.int/_data/assets/pdf_file/0003/79374/ E88105.pdf), June 2002. Accessed 15 May 2014.

3. Global Polio Eradication Initiative. Key countries (http:// www.polioeradication.org/Keycountries.aspx). Accessed 6 December 2015.

4. Slater PE, et al. Poliomyelitis outbreak in Israel in 1988: a report with two commentaries. Lancet. 1990; 335: 1192-1195.

5. Swartz TA. The epidemiology of polio in Israel. An historical perspective. Israel Center for Disease Control (ICDC) Publication 317. Tel Aviv, Israel: Dyonon Publishing, 2008.

6. Shulman LM, et al. Molecular epidemiology of silent introduction and sustained transmission of wild poliovirus type 1, Israel 2013. Eurosurveillance 2014; 19: 20709.

7. Anis E, et al. Insidious reintroduction of wild poliovirus into Israel. Eurosurveillance 2013; 18: 20586.

8. Kopel E, Kaliner E, Grotto I. Lessons from a public health emergency-importation of wild poliovirus to Israel. New England Journal of Medicine 2014; 371: 981-983.

9. Israel Control of Disease Center (ICDC). Health 2013. Ministry of Health (http://www.health.gov.il/publicationsfiles/health2013.pdf), 1 February 2014. Accessed 15 May 2014.

10. Rainey $\mathbf{J}$, et $\boldsymbol{a l}$. Reasons related to non-vaccination and under-vaccination of children in low and middle income countries: finding from systematic review of the published literature 1999-2009. Vaccine 2011; 29: 8215-8221.

11. Brown KF, et al. Factors underlying parental decisions about combination childhood vaccinations including MMR: a systematic review. Vaccine 2010; 28: 4235-4248.

12. Topuzoglu A, et al. Assessment of socio-demographic factors and socio-economic status affecting the coverage of compulsory and private immunization services in Istanbul, Turkey. Public Health 2005; 119: 862-869.

13. Wei F, et al. Identification and characteristics of vaccine refusers. BMC Pediatrics 2009; 9: 18-26.

14. Smith PG, Chu SY, Barker LE. Children who have received no vaccine: who are they and where do they live? Pediatrics. 2004; 114: 187-195.

15. Central Bureau of Statistics. Population by population group (http://www.cbs.gov.il/publications14/yarhon0514/pdf/b1.pdf). Accessed 9 June 2014.

16. National Council for the Child. Children in Israel 2013 (http://www.children.org.il/files/fle/SHNATON/\%20\% 202013.pdf), December 2013. Accessed 4 June 2014.

17. Ministry of Health. National vaccination coverage (http://www.health.gov.il/subjects/vaccines/two_drops/ pages/vaccinationcoverage.aspx), December 2013. Accessed 20 January 2014.

18. Central Bureau of Statistics. Characterization and classification of geographical units by the socio-economic 
level of the population 2008 (http://www.cbs.gov.il/webpub/pub/text_page_eng.html?publ $=100 \&$ CYear $=2008 \&$ CMonth=1). June 2013. Accessed 5 February 2014.

19. Kalter-Leibovici O, et al. Obesity among Arabs and Jews in Israel: a population study. Israel Medical Association Journal 2007; 9: 525-530.

20. Daoud N. Challenges facing minority women in achieving good health: voices of Arab women in Israel. Women Health 2008; 48: 145-166.

21. Zamir CS, et al. Measles outbreaks affecting children in Jewish ultra-orthodox communities in Jerusalem. Epidemiol Infect 2008; 136: 207-214.

22. Central Bureau of Statistics. Geographic distribution and demographic, social and economic characteristics of ultra-orthodox Jewish population in Israel 19962001 (http://www.cbs.gov.il/www/pulications/int_ulor. pdf), July 2004. Accessed 6 December 2015.

23. Zamir CS, et al. The national childhood immunization registry in Israel. Israel Medical Association Journal 2011; 12: 296-300.

24. Metzger AM, Friedrich PK, Kuint J. To vaccinate or not to vaccinate-that is the question: why are some mothers opposed to giving their infants hepatitis B vaccine? Vaccine 2005; 23: 1941-1948.

25. Oyserman D, Coon HM, Kemmelmeir M. Rethinking individualism and collectivism: evaluation of theoretical assumption and meta-analysis. Psychological Bulletin 2002; 128: 3-72.

26. Green E, Deschamps JC, Paez D. Variation of individualism and collectivism within and between 20 countries. A typological analysis. Journal of Cross-Cultural Psychology 2005; 36: 321-339.

27. Freeman MA. Demographic correlates of individualism and collectivism: a study of social values in Sri Lank. Journal of Cross-Cultural Psychology 1997; 28: 321-341.

28. Marshall R. Variances in levels of individualism across two cultures and three social classes. Journal of Cross-Cultural Psychology 1997; 28: 430-495.

29. Luyten J, et al. Kicking against the pricks: vaccine skeptics have a different social orientation. European Journal of Public Health 2014; 24: 310-314.

30. Velan B, et al. Individualism, acceptance and differentiation as attitude traits in the public's response to vaccination. Human Vaccines \& Immunotherapeutic 2012; 8: 1272-1282.

31. Kaliner E, et al. Silent reintroduction of wild-type poliovirus to Israel, 2013 - risk communication challenges in an argumentative atmosphere. Eurosurveillance 2014; 19: 20703

32. Avesld L. Mass vaccination programmes and the value of respect for autonomy. Bioethics 2008; 22: 245-257. 